Abstract P139 Table 1 Descriptive statistics of individual subgroups of the study

\begin{tabular}{|l|c|l|l|}
\hline \multicolumn{2}{|c|}{} & \multicolumn{2}{c|}{ Mean \pm SD } \\
\hline Group Total & $\mathrm{n}=58$ & $512.30 \pm 89.40$ & $511.70 \pm 87.90$ \\
\hline Gender; Male & $\mathrm{n}=30$ & $584.24 \pm 49.43$ & $583.58 \pm 48.84$ \\
\hline Gender; Female & $\mathrm{n}=28$ & $435.30 \pm 48.60$ & $434.78 \pm 43.22$ \\
\hline Age; 18-39 & $\mathrm{n}=41$ & $525.00 \pm 85.40$ & $523.0 \pm 85.2$ \\
\hline Age; 40-60 & $\mathrm{n}=18$ & $479.80 \pm 95.20$ & $483.70 \pm 92.80$ \\
\hline CPEFmax & $\mathrm{n}=58$ & $522.60 \pm 89.60$ & $523.80 \pm 88.80$ \\
\hline
\end{tabular}

with the different interfaces. Analysis of each interfaces' maximal effort $\left(\mathrm{CPEF}_{\max }\right)$ indicated no significant differences (CI $95 \%, p=0.943)$. Randomised sequence data was analysed, where it concluded that there was no significant influence of interface sequencing on the Results $(\mathrm{CI}=95 \%, \mathrm{p}=0.671)$.

Conclusion The study's Results support the hypothesis, suggesting interchangeability of both interfaces. This now offers a platform for further study in the viability of facemask CPEF within the clinical setting, as well as providing a standardised protocol and CPEF reference values.

\section{P140 HYPOXIC CHALLENGE TESTING IN MOTOR NEURONE DISEASE}

IJ Cliff, N Mustfa, H Stone. University Hospitals of North Midlands, Stoke-on-Trent, UK

\subsection{6/thoraxjnl-2017-210983.282}

Introduction Respiratory muscle weakness is a feature of motor neurone disease (MND), develops insidiously and presents with subtle symptoms. It can be difficult to assess in MND patients who, as a result, may be at risk of desaturation at altitude. Hypoxic challenge tests (HCT) can identify patients who would benefit from in-flight oxygen, but evidence as to which patients should be referred is lacking. The aim of this study was to identify factors that may predict the need for in-flight oxygen in this group of patients where maintaining their independence for as long as possible is paramount.

Methods 81 consecutive HCT's in 53 male, 28 female patients, and the contemporaneous assessments for respiratory muscle weakness on patients with MND. Data from patients requiring in-flight oxygen according to the HCT was compared to data from patients who did not, in accordance with the BTS Guidance for Air Travel 2011.

Results The median patient age of patients who passed the HCT was 62 years; those that failed the HCT were significantly older with a median age of 68 years $(p=0.009)$. There was a significant difference in baseline $\mathrm{PaO}_{2}$ and $\mathrm{PaCO}_{2}$ between the groups as shown in Table 1; patients who passed the HCT had higher baseline $\mathrm{Pa} 02$ and lower $\mathrm{PaC0} 2$ $(10.4 \mathrm{kPa}$ and $5.3 \mathrm{kPa}$ versus $9.3 \mathrm{kPa}$ and $6.2 \mathrm{kPa}$ respectively $\mathrm{p}=0.0001$ and 0.0014 ). No other parameter, including BMI, smoking history, or physiological measurement including SNIP, or spirometry, could predict the outcome of the HCT.

Conclusions Although MND patients that are likely to fail a HCT have a higher baseline $\mathrm{C} 02$, a threshold $\mathrm{C} 02$ value that could identify patients needing in-flight oxygen was not determined. We recommend that the safest approach is to refer all patients with MND that intend to fly for HCT assessment until more evidence-based data is available, which is the current practice at this regional centre.

Abstract P140 Table 1 Demographic data for the 2 patient groups - those that pass and those that fail the HCT

\begin{tabular}{lll}
\hline & Pass & Fail \\
\hline Number of Tests & 60 & 21 \\
Age (Years) & $62(57.5-68.0)$ & $68(61.5-71.8) * *$ \\
BMI & $24.5(21.7-28.0)$ & $24.9(21.5-28.1)$ \\
Pack years & $8.5(0.4-15.5)$ & $1.5(0.0-31.0)$ \\
$\mathrm{pH}$ & $7.43(7.41-7.45)$ & $7.42(7.40-7.43)$ \\
PaC02 kPa & $5.3(5.1-5.8)$ & $6.2(5.4-6.7) * *$ \\
Pa02 kPa & $10.4(9.5-11.1)$ & $9.4(8.8-9.7) * *$ \\
HCO3 & $26.4(25.1-27.7)$ & $27.8(26.9-30.8) * *$ \\
BE & $2.5(0.9-3.8)$ & $4.5(2.8-6.1)^{* *}$ \\
Sa02 & $95(95.0-95.0)$ & $96(95.0-96.0)$ \\
SNIP (cmH20) & $35.0(25.0-49.3)$ & $30.5(21.5-54.0)$ \\
FEV1 (L) & $2.1(1.5-2.7)$ & $1.5(0.77-2.79)$ \\
FEV1\% Predicted & $71.00(51-93)$ & $53.00(42-96)$ \\
FVC (L) & $2.7(1.81-3.27)$ & $1.8(1.07-3.08)$ \\
FVC\% Predicted & $69(46-87)$ & $71(54-89)$ \\
FEV1/FVC & $83(77.5-90.0)$ & $77(65.8-84.9)$ \\
\hline
\end{tabular}

** denotes significant difference between the groups with a $p$ value $<0.01$

\section{P141 PULMONARY FUNCTION TEST PHYSIOLOGY AND PROGRESSION IN DIFFUSE IDIOPATHIC PULMONARY NEUROENDOCRINE CELL HYPERPLASIA (DIPNECH)}

1) Barlow, ${ }^{2} \mathrm{D}$ Ryan, ${ }^{3} \mathrm{~W}$ Mansoor, ${ }^{3} \mathrm{M}$ Howell, ${ }^{4} \mathrm{~N}$ Clayton, ${ }^{4} \mathrm{R}$ Niven. ${ }^{1}$ University of Manchester, Manchester, UK; ${ }^{2}$ Beaumont Hospital, Respiratory Department, Dublin, Ireland; ${ }^{3}$ The Christie NHS Foundation Trust, Manchester, UK; ${ }^{4}$ North West Lung Centre, University Hospital South Manchester, Manchester, UK

10.1136/thoraxjnl-2017-210983.283 
Introduction Diffuse idiopathic pulmonary neuroendocrine cell hyperplasia (DIPNECH) is a rare condition characterised by a generalised proliferation of pulmonary neuroendocrine cells within the respiratory epithelium. Current literature is limited, in particular little is known of its affects on pulmonary function both at the time of diagnosis and prospectively, though it is recognised to cause small airway obstruction.

Objective The aim of this study was to characterise pulmonary function, both at baseline and to also define the change in pulmonary function over time in patients with DIPNECH.

Methods Retrospective analysis of pulmonary function data for patients with a histological diagnosis of DIPNECH was performed. At baseline, pulmonary function was characterised as either obstructive, small airways obstruction, restrictive, mixed (obstructive and restrictive) or normal. Baseline gas transfer (DLCO) and lung volume data was also described. FEV1 was used as the main measure of pulmonary function, and simple linear regressions were created for patients with longitudinal data. This then allowed basic statistical analysis of the change in FEV1 compared to the predicted change.

Results 17 patients ( $82 \%$ female), with a mean age of 59, were included. All had pulmonary function data at baseline and $9(53 \%)$ had prospective data. Baseline pulmonary function was predominantly obstructive in nature with $6(35 \%)$ having classical obstruction, and 7 (41\%) small airways obstruction alone with a normal FEV1/FVC ratio, the remaining 4 having either normal $(n=3,23 \%)$ or mixed $(n=1,6 \%)$ physiology. The mean FEV1 at baseline was $81.6 \%$, and a statistically significant difference was present between mean measured and predicted FEV1 values for the cohort $(p=0.02)$. Mean DLCO $(n=13)$ was mildly decreased at $84.6 \%$ predicted however corrected to normal with volume. Lung volume data $(n=8)$ where available was normal, except in two patients $(12 \%)$ who had significantly increased residual volume. Patients with longitudinal data $(\mathrm{n}=9,53 \%)$ predominantly showed a stable pattern of obstruction with minimal decline. Two patients (12\%) did have a significantly increased decline compared to predicted values.

Conclusion Patients with DIPNECH typically have a stable degree of fixed obstruction, however exceptions to this will be seen in patients with a more progressive disease.

\section{P142 HYPOXIC CHALLENGE TEST (HCT) FOR IN-FLIGHT OXYGEN ASSESSMENTS CAN BE AVOIDED IN PATIENTS WITH LUNG DISEASE AND LOW RESTING PAO2}

R Peat, F Frost, K Waldron, J Furlong, D Russell, D Wat. Liverpool Heart and Chest Hospital, Liverpool, UK

\subsection{6/thoraxjnl-2017-210983.284}

Introduction Hypoxic challenge test consisting of breathing $15 \% \mathrm{FiO} 2$ for $20 \mathrm{~min}$ with blood gas measurements is recommended by BTS guidelines for the assessment of the requirement for in-flight oxygen. FEV1 and SpO2 have been demonstrated to be poor predictors of desaturation with no established reliable upper limit of $\mathrm{PaO} 2$, above which patients will not desaturate. We investigated whether there were lowerlimit thresholds, beyond which oxygen is always required and hence HCT can be avoided.

Methods Retrospective analysis of all hypoxic challenge tests conducted at our centre between 2010 and 2017 was undertaken. Baseline demographics, diagnosis and contemporaneous lung function data was recorded. HCT was performed as per BTS guidance and included baseline resting blood gas followed by a repeat after $20 \mathrm{~min}$ inspiring $15 \% \mathrm{FiO} 2$. If $\mathrm{PaO} 2$ was $<6.65 \mathrm{kPa}$ or $\mathrm{SpO} 2<85 \%, 2 \mathrm{~L}$ oxygen via nasal cannulae was applied and a repeat blood gas performed to confirm $\mathrm{PaO} 2 \geq 6.65 \mathrm{kPa}$.

Results HCT was performed on 170 occasions during the study period. COPD was the underlying diagnosis in 110 (64.7\%) of tests, ILD in 40 (23.5\%) and CF (13, 11.8\%). Average age (median [range]) was 67.5 years [49.1-83.8] COPD, 67 [52.3-83.3]ILD, 32.5 [19.1-66.8]CF. Lung function (FEV1\%pred) was 49.7[21-115]COPD, 71.6 [31-124]ILD, 36.5[23-65]CF. Following HCT, in-flight oxygen was recommended in $99(58.2 \%)$ patients all of whom were recommended $21 / \mathrm{min}$. A threshold of $<7.55 \mathrm{Kpa}$ on resting blood gas was $100 \%$ predictive for requirement of in-flight oxygen and a threshold of $<8 \mathrm{kPa}$ was $97.9 \%$ predictive. Incorporating the $<7.55 \mathrm{kPa}$ and $<8 \mathrm{kPa}$ thresholds into clinical practice by proceeding straight to 21 oxygen could negate the need for HCT in $20.6 \%$ and $43.9 \%$ of cases respectively.

Conclusion HCT is a useful tool for assessing the need for inflight oxygen in lung diseases but is a resource heavy test and requires multiple blood samples taken from patients. Our data suggests that there are lower-limit thresholds for resting $\mathrm{PaO} 2$ beyond which HCT can be avoided in a significant proportion of patients.

\section{P143 USING BIG DATA TO INVESTIGATE PHYSIOLOGY: RETENTION OF CO2 DOES NOT IMPACT THE OXYGEN- HAEMOGLOBIN DISSOCIATION CURVE OF CRITICALLY ILL ADULTS}

${ }^{1} \mathrm{~N}$ Rosculet, ${ }^{1} \mathrm{R}$ Samata, ${ }^{1} \mathrm{~A}$ Dixit, ${ }^{2} \mathrm{~S}$ Harris, ${ }^{2} \mathrm{NS}$ MacCallum, ${ }^{2} \mathrm{DA}$ Brearley, ${ }^{3} \mathrm{PJ}$ Watkinson, ${ }^{4} \mathrm{~A}$ Jones, ${ }^{5} \mathrm{~S}$ Ashworth, ${ }^{4} \mathrm{R}$ Beale, ${ }^{5} \mathrm{SJ}$ Brett, ${ }^{3} \mathrm{JD}$ Young, ${ }^{2} \mathrm{M}$ Singer, ${ }^{1} \mathrm{C}$ Summers, ${ }^{1} \mathrm{~A}$ Ercole. ${ }^{1}$ Department of Medicine, University of Cambridge School of Clinical Medicine, Cambridge, UK; ${ }^{2}$ Bloomsbury Institute of Intensive Care Medicine, University College London, London, UK; ${ }^{3}$ Nuffield Department of Clinical Neurosciences, University of Oxford, Oxford, UK; ${ }^{4}$ Department of Intensive Care Medicine, Guy's and St Thomas' Hospital NHS Foundation Trust, London, UK; ${ }^{5}$ Centre for Perioperative Medicine and Critical Care Research, Imperial College Healthcare NHS Trust, London, UK

\subsection{6/thoraxjnl-2017-210983.285}

Introduction Since its initial description in 1904, the oxygenhaemoglobin dissociation curve (ODC) has been well described under physiological conditions. ${ }^{1,2}$ However, the impact of pathology has been less well characterised, with most data arising from small clinical studies of anaesthetized adults/ patients $(<100$ subjects), or experimentally-induced hypoxaemia/hypercapnia. Routinely collected clinical data, including arterial blood gas analyses, are now available from many thousands of critically ill patients. We sought to investigate the impact of $\mathrm{pCO}_{2}$ on the ODC of critically ill adults, and hypothesised that $\mathrm{pCO}_{2}$ would not significantly alter the relationship between $\mathrm{pO}_{2}$ and haemoglobin saturation.

Methods Data was extracted from the National Institute for Health Research Critical Care Health Informatics Collaborative (NIHR ccHIC). Statistical analysis was undertaken on 399000 blood gases from 13942 patients, using R version 3.4.0. After data cleaning, the predicted oxygen saturation for each arterial blood gas sample was calculated using both the Severinghaus ${ }^{1}$ and Dash, Kroman and Bassingthwaighte ${ }^{2}$ equations. Non-linear regression modelling was undertaken to construct ODCs based on both the predicted and observed data, 